\title{
Microwave Planar Sensor for Determination of the Permittivity of Dielectric Material
}

\author{
Mohd Khairy Ismail, Zahriladha Zakaria, Nornikman Hassan, Sam Weng Yik, Mohd Mawardy \\ Abdullah \\ Centre for Telecommunication Research and Innovation (CeTRI), \\ Faculty of Electronics and Computer Engineering (FKEKK), \\ Universiti Teknikal Malaysia Melaka (UTeM), Durian Tunggal, Melaka, Malaysia
}

\begin{tabular}{|c|c|}
\hline Article Info & ABSTRACT \\
\hline Article history: & This paper proposed a single port rectangular microwave resonator sensor. \\
\hline Received Aug 18, 2018 & $\begin{array}{l}\text { This sensor operates at the resonance frequency of } 4 \mathrm{GHz} \text {. The sensor } \\
\text { consists of micro-strip transmission line and applied the enhancement }\end{array}$ \\
\hline Revised Oct 26, 2018 & method. The enhancement method is able to improve the return loss of the \\
\hline Accepted Nov 12, 2018 & $\begin{array}{l}\text { sensor, respectively. Plus, the proposed sensor is designed and fabricated on } \\
\text { Roger } 5880 \text { substrate. Based on the results, the percentage of error for the }\end{array}$ \\
\hline evwords & \\
\hline
\end{tabular}

Microwave sensor

Pushing effect

Q-factor

Return loss

Copyright $@ 2018$ Institute of Advanced Engineering and Science. All rights reserved.

\section{Corresponding Author:}

Mohd Khairy Ismail,

Centre for Telecommunication Research and Innovation (CeTRI),

Faculty of Electronics and Computer Engineering (FKEKK),

Universiti Teknikal Malaysia Melaka (UTeM),

Durian Tunggal, Melaka, Malaysia.

Email: 1sntl@ccu.edu.tw

\section{INTRODUCTION}

In recent years, material characterization techniques have been introduced. Many techniques are available to measure the real part of the complex permittivity or dielectric constant. In a material, there is the mixture of different size of molecules. Then, from the structure of molecule inside the material, the permittivity can be determined. The response of material to the electrical signal depends on the permittivity of the material. Generally, the accurate determination of the permittivity is an important task for microwave or radio frequency circuit design, antenna design and of course to the microwave engineering [1-4]. Furthermore, the changes of dielectric properties of material or material characterization are widely used in many fields such as food industry, quality control, bio-sensing or medical industry [5-8].

There are two types of methods that can be used to measure the dielectric through a microwave technique which are non-resonant and resonant methods. Resonant method acquires high accuracy compared to non-resonant method; the reason of choosing resonant method instead of non-resonant method. Then, through resonant method, the material under test (MUT) is introduced to the resonator; change the electromagnetic boundaries of the resonator. Meanwhile, the non-resonant method is used to get the electromagnetic properties of the material over a frequency range.

The resonator is usually applied as an accurate instrument for electromagnetic properties of a material like complex permittivity, permeability and the resistance for microwave frequencies compared to non-resonant. Furthermore, it is widely being used for low-loss, small size sample and sample with irregular 
shape due to its high accuracy. The high-quality factor (Q-factor) of the resonator causes a high sensitivity device where it can be used to sense the difference in physical quantities; depends on the complex permeability of MUT [9-11].

\subsection{Perturbation Technique}

The most frequent technique to measure the complex permittivity of lossy material is known as the perturbation technique. This technique has been used for measuring the electromagnetic characteristics between the empty and partially loaded MUTs [12]. When a small piece of dielectric material (MUT) is introduced into the resonant cavity, the resonance frequency is shifted by a small amount [13]. Plus, the selectivity of the cavity is lowered. These effects are commonly used in the measurement of properties of the material; the relation between the shifted of resonance frequencies, selectivity and the permittivity of the sample.

The MUTs is placed at a specific location on the resonator where the electric field (e-field) is at a maximum condition. Then, the insertion of the MUTs within the cavity causes the effect of perturbation to the overall circuit. The perturbation causes a shifting of the resonance frequency and a decrease in the unloaded Q-factor. The cavity response in the perturbation is related to the dielectric properties of the tested material through the cavity perturbation theory. Based on the theory of perturbation, it is anticipated that the fractional change in the resonant frequency has increased with the increasing of the dielectric constant of the MUTs [14]. The resonance frequency is shifted to the lower frequency when the MUTs are being placed on the resonator sensor. This is because of the maximum electric field of the resonator sensor when it's perturbed to the sample.

\subsection{Reflection Method}

Reflection methods is a method which able to measure only one of the parameters, either the permittivity or the permeability. In this project only, the permittivity parameter is measured. The electromagnetic waves are directed to the MUT, and then the properties of the sample is detected from the reflection coefficient that occurs in the defined reference plane. To apply the reflection method in the measurement of the dielectric property, one port resonator is mainly used. The single port capability to transmit the signal in one port, then, the received signal is reflected from the MUT to the same port it transmits before. The measured $\mathrm{S}$-parameter is $\mathrm{S}_{1,1}$ or $\mathrm{S}_{2,2}$. The dynamic range of reflection measurements is limited by the directivity of the measurement port.

The one port calibration is compulsory to improve the accuracy and sensitivity of the measurement of the system. Through the calibration, it can measure and remove the three systematic error terms in the measurement of the system; the directivity, source match and also the reflection tracking. The simple calibration is enough to be conducted [15]. In Figure 1, it shows the graph of S-parameter, S1, 1. The value of the $\mathrm{S} 1,1$ can be determined by using the equation 1.1; for determining the half-power width. In the equation, the $\mathrm{S} 1,1 \mathrm{~b}$ is the base line of the resonance for $\mathrm{S} 1,1$ value. While, the $S 1,1_{\mathrm{fo}}$ is the resonance frequency value for the S1, 1 [16]. Besides, from Figure 1 also the Q-factor of the resonator can be determined based on the bandwidth and the resonant frequency which is:

$$
S_{1,1 \Delta f}=10 \log \left(\frac{10^{\frac{s 1,1 b}{10}}+10^{\frac{s 1,1 f 0}{10}}}{2}\right)(d B)
$$

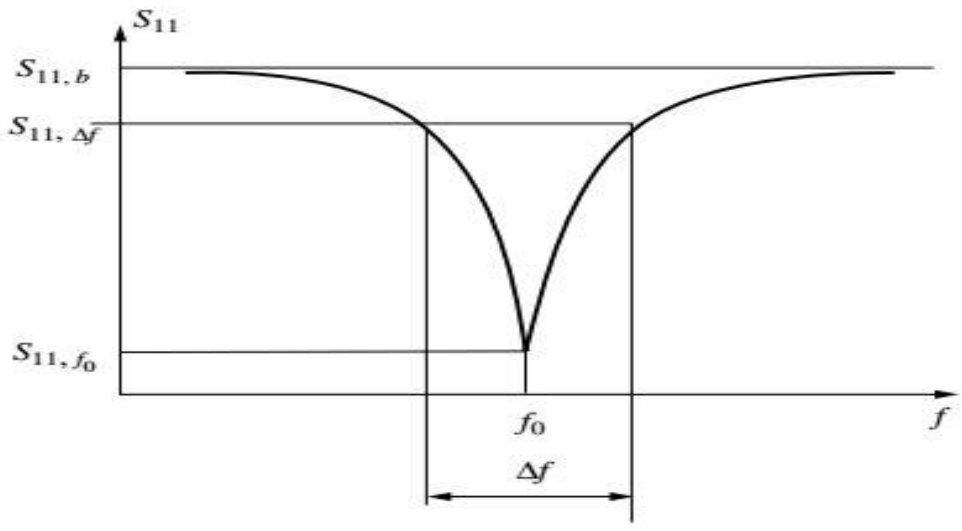

Figure 1. S-parameter for the reflection method 


\section{GEOMETRY DESIGN \& MATHEMATICAL ANALYSIS}

Figure 2 shows the modified Edge Couple Split Ring Resonator (EC-SRR) structure. The dimension of the larger structure is $7.2 \mathrm{~mm}$ length (LSUB) x $7.2 \mathrm{~mm}$ width (WSUB) while the dimension of the smaller SRR is only $4.32 \mathrm{~mm}$ length $\mathrm{x} 4.32 \mathrm{~mm}$ length. The dimension of WSSR 1 and WSSR2 is $10.13 \mathrm{~mm}$ and 7.60 $\mathrm{mm}$, respectively.

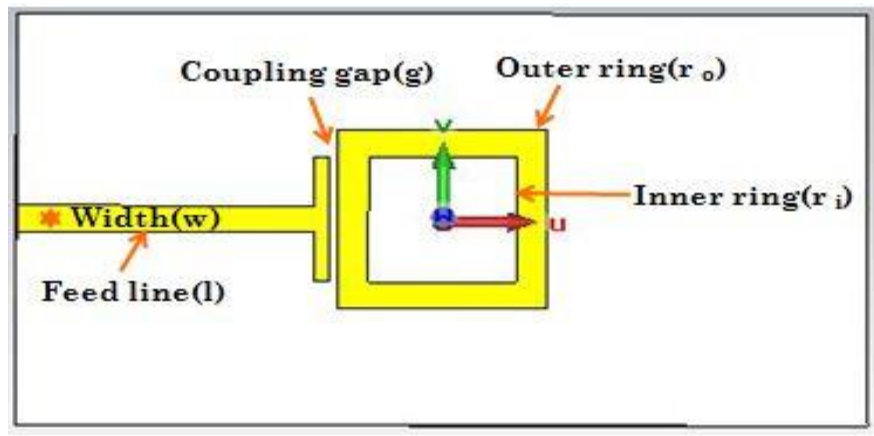

Figure 2. Schematic diagram of the proposed rectangular resonator sensor

Equations 2 to 18 are shown below to design a micro-strip ring resonator, the principle that can be used to enable the changes of effective permittivity if any dielectric material is placed on the substrate surface; cause the changes in the resonant frequency. The resonant frequency is determined by using the equation of $f=\frac{1}{2 \pi \sqrt{L C}}$, where inductance, $L=\mu_{o} R_{m}\left(\ln \frac{8 R_{m}}{h+w}-0.5\right), R_{m}$ is main radius of the ring, $h$ is height of the substrate, and $w$ is the width of the feed-line. The capacitor, $C$ can affect the resonant frequency and it can be determined by $C=\frac{\varepsilon_{r o} \varepsilon_{r} A}{d}$, with $A$ is area of the gap, $d$ is Distance of the gap, $\varepsilon_{r}$ is relative permittivity of dielectric presents between the plates. To calculate the feed-line dimension and coupling the following equations are used, where the feed-line width is $A=\frac{z_{0}}{60} \sqrt{\frac{\varepsilon_{r}+1}{2}}+\frac{\varepsilon_{r}+1}{\varepsilon_{r}-1}\left(0.23+\frac{0.11}{\varepsilon_{r}}\right)$, with $\frac{w}{d}=$ $\frac{8 e^{A}}{e^{2 A} A_{-}}$, which is $d$ is the thickness of the substrate with Feedline length, $\iota=\frac{\lambda g}{4}$, The coupling gap, $\lambda g=$ $\frac{c}{f \sqrt{\varepsilon_{e f f}} .} \therefore \lambda \mathrm{g}$ is the wavelength at the given frequency. The effective permittivity, Eff.permittivity, $\varepsilon_{e}=$ $\frac{\varepsilon_{r}+1}{2}+\frac{\varepsilon_{r}-1}{2}\left(\frac{1}{\sqrt{1+\frac{12 d}{w}}}\right)$ while the coupling gap, Coupling gap, $\Delta L=0.421 d\left(\frac{\varepsilon_{e f f}+0.3}{\varepsilon_{e f f^{-}}-0.258}\right)\left(\frac{\frac{w}{d}+0.262}{\frac{w}{d}+0.818}\right)$.

The micro - strip resonator sensor is a close-loop transmission line. To form the resonant frequency, the power is capacitive coupled through the feed-line and the gap between them. So, the resonant frequency happens when the mean circumstances of the ring are equal to the integral of the guided wavelength. $2 \pi r=n \lambda g$ for $\mathrm{n}=1,2,3 \ldots$, with the Frequency dependent, $\lambda g=\frac{\lambda}{\sqrt{\varepsilon_{e f f}}}=\frac{1}{\sqrt{\varepsilon_{e f f}}} \frac{c}{f}$, which is the $f_{o}=$ $\frac{n c}{2 \pi r \sqrt{\varepsilon_{e f f}}}$, with $r$ is ring radius, $n$ is $1,2,3 \ldots, c=3 \times 10^{8} \mathrm{~m} / \mathrm{s}$ (speed of light) and $\varepsilon_{e f f}$ : is Effective permittivity of the substrate.

Explaining research chronological, including research design, research procedure (in the form of algorithms, Pseudocode or other), how to test and data acquisition [1], [3]. The description of the course of research should be supported references, so the explanation can be accepted scientifically [2], [4].

$\therefore$ Decreasing in gap cause the gap capacitance increases and make the coupling tighter. Then the increase in gap capacitance effect the resonator frequency; lower frequency. This circumstance is known as 'pushing effect'.

The effective permittivity regarding the resonant frequency, $f_{c}$ with $\varepsilon_{e f f}=\left(\frac{n c}{2 \pi f_{c} r_{m}}\right)^{2}$, with Outer radius, $R_{o}=r+\frac{w}{2}$, Inner radius, $R_{i}=r-\frac{w}{2}$, Quality factor, $Q=\frac{2 f_{c}}{B W}, f_{c}$ is resonant frequency and $\mathrm{BW}$ is Bandwidth of the resonant frequency.

The length of substrate, $\mathrm{L}_{\mathrm{g}}$ can be determined by: Length of substrate, $L g=2 l+2 \Delta L+2 R_{o}$. While, for the width of the substrate, Wg: Width of substrate, $W g=2 R_{o}+\frac{\lambda g}{4}$. 
Through this project of microwave sensor, the numerical result will be used for circuit design in the CST software. The parameters being used are illustrated in Table 1.

Table 1. Parameters in Designing the Single Port Rectangular Resonator Sensor

\begin{tabular}{lc}
\hline \multicolumn{1}{c}{ Parameter } & Design Value \\
\hline Substrate: Roger 5880 & Freq: $4 \mathrm{GHz}$ \\
Substrate thickness & $0.787 \mathrm{~mm}$ \\
Substrate permittivity, $\varepsilon_{r}$ & 2.2 \\
Length of substrate $(L g)$ & $68.12 \mathrm{~mm}$ \\
Width of substrate $(W g)$ & $38.69 \mathrm{~mm}$ \\
Feed-line length $(l)$ & $25.35 \mathrm{~mm}$ \\
Outer ring $\left(r_{o}\right)$ & $8.4 \mathrm{~mm}$ \\
Inner ring $\left(r_{i}\right)$ & $5.9 \mathrm{~mm}$ \\
Gap $(g)$ & $0.62 \mathrm{~mm}$ \\
Width of feed-line $(w)$ & $2.5 \mathrm{~mm}$ \\
\hline
\end{tabular}

\section{RESULTS AND ANALYSIS}

After simulation work done, it shows that the antenna bandwidth had been enhanced by the addition of the modified EC-SRR. An improvement of the bandwidth is $727 \mathrm{MHz}$, increase from $0.645 \mathrm{GHz}$ (Design $A$ ) to $1.372 \mathrm{GHz}$ (Design $D$ ). The return loss also increases but not gives the significant effect to the antenna design.

\subsection{Simulated Result}

The single port rectangular resonator sensor with the resonance frequency of $4 \mathrm{GHz}$ is designed. The structure dimension of the resonator sensor is calculated based on the mathematical analysis. While, three parameters are measured through the sensor; the resonant frequency, Q-factor and the return loss with five different samples and permittivity; air, Roger 5880, Roger 4350, FR-4 and Roger 3010.

The sensor is constructed by using the Roger 5880 with the thickness of $0.787 \mathrm{~mm}$ and loss tangent of 0.009. The basic rectangular resonator sensor is demonstrated in Figure 3. But, based on the S1, 1 result, it shows the sensor operates on $3.992 \mathrm{GHz}$ and has a large return loss which is $-6.4881 \mathrm{~dB}$; the targeted return loss is less than $-10 \mathrm{~dB}$.

Although the basic resonator sensor able to shows a good performance in the resonant frequency and in the strength of the electromagnetic field, the basic design needs a modification. Thus, a modification in the design is proposed to improve the return loss of the resonator sensor. The enhancement method is introduced in the design to overcome the problem of low performance in the resonator due to the large return loss. Figure 4 shows the structure of the rectangular sensor with the enhancement method.

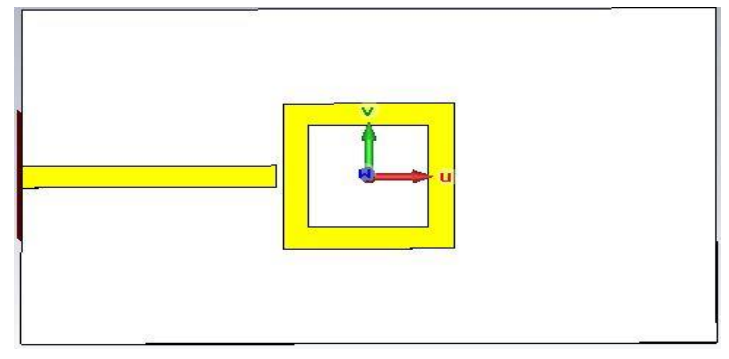

Figure 3. The basic rectangular resonator sensor

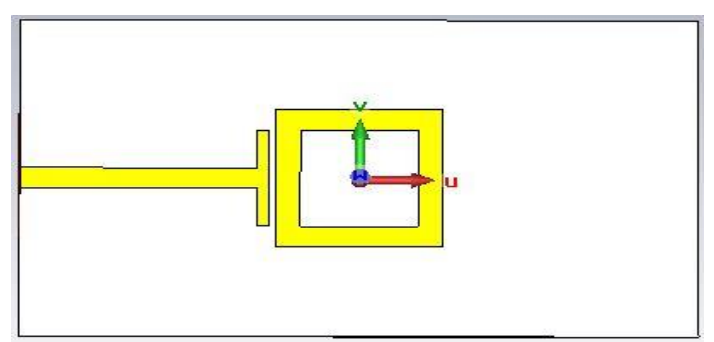

Figure 4. The structure of proposed resonator sensor

The performance of the proposed resonator sensor with the enhancement method is measured based on the $\mathrm{S} 1,1$ signal due to the usage of the reflection method. The bandwidth of the $\mathrm{S} 1,1$ is gathered at $-3 \mathrm{~dB}$ from the base line of the resonance of S1, 1; refer to Figure 5. So, the resonance frequency is occurring at $3.992 \mathrm{GHz}$ while the bandwidth of the $\mathrm{S} 1,1$ is $0.070678 \mathrm{GHz}$. The Q-factor of the proposed resonator sensor in the simulation is 113 .

The MUTs are placed in a maximum electric field (e-field) region as indicated in Figure 5 and Figure 6. Thus, the resonator sensor produces a reduction in the Q-factor as well as the reduction in the resonant frequency as associated with the loss of the material. Then, the resonator sensor can be used to 
measure the dielectric properties of the MUTs by using the perturbation method; based on the shifting of the resonance frequency.

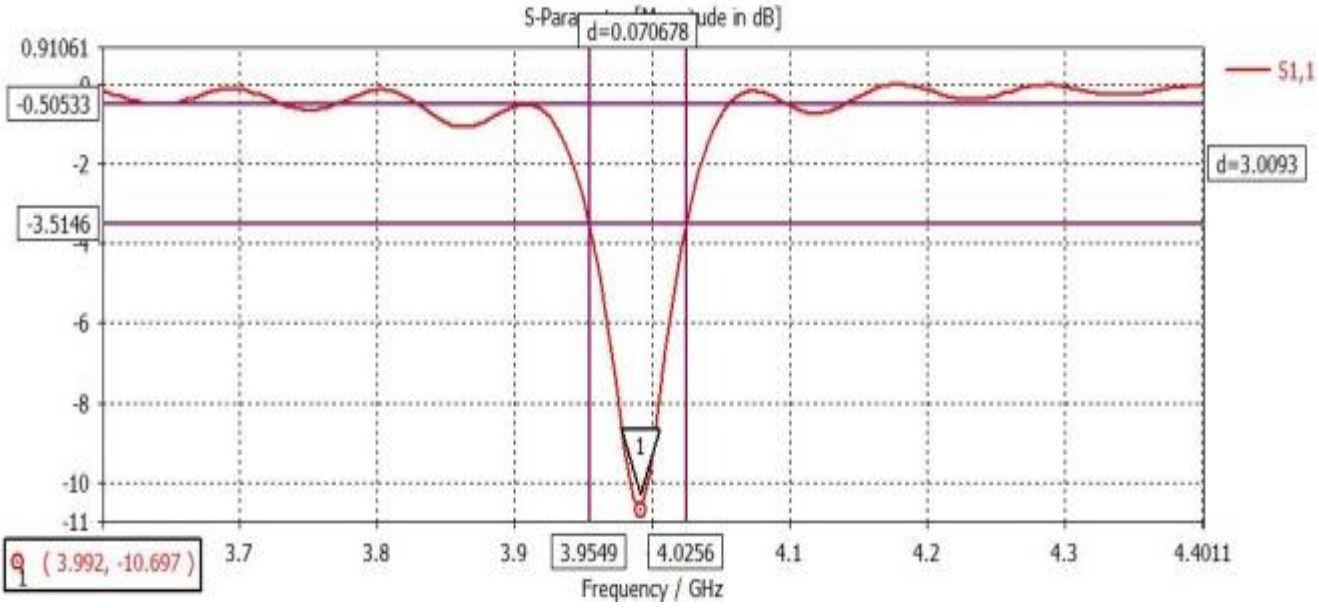

Figure 5. The $S_{1,1}$ graph of the proposed design

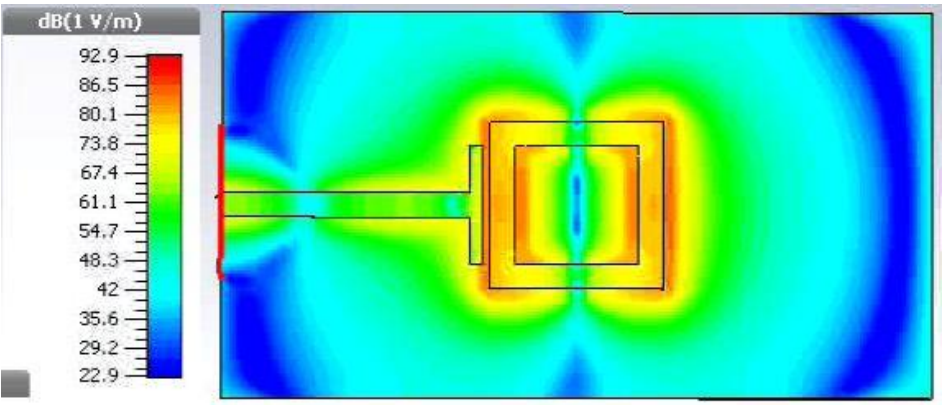

Figure 6. The e-field in the resonator sensor

Figure 7 shows the overlay sample on the resonator sensor. In Figure 8, it shows the changes in the resonant frequency after different types of material is placed on the resonator sensor through simulation in CST software, the frequency is shifted. Four samples have been tested like FR-4, Roger 5880, Roger 4350 and Roger 3010. The resonant frequency with the sample of air refers to the without sample situation. Besides, based on Figure 8 also, the frequency is shifted to the lower frequency when the overlay sample is used. This is because of the maximum electric field of the resonator when it is perturbed to the sample. The return loss level $(\mathrm{dB})$ also shows a variation when the sample is applied to the resonator sensor due to the effective dielectric constant.

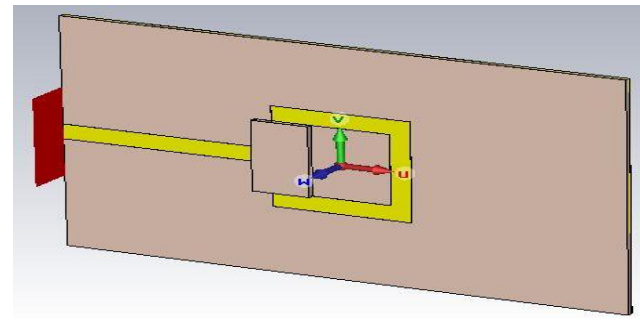

Figure 7. The overlay sample on the resonator sensor 


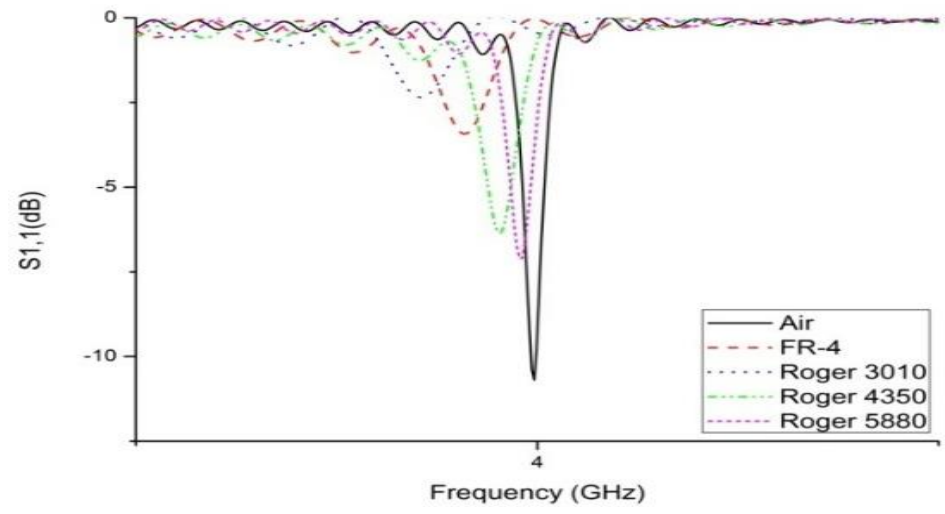

Figure 8 . The changes of resonant frequency with different overlay samples in simulation

Based on both data representations in Figure 9, the sensitivity of the sensor is represented in terms of relative shift in resonance frequency with the permittivity of MUTs. It's concluded that the higher permittivity of the samples causes the higher the percentage of frequency shifting. Roger 5880 has the lowest percentage which is $2.6 \%$, followed by Roger 4350, $8.4 \%$, FR-4 $16.8 \%$ and Roger 3010, $27.6 \%$. Where the permittivity of Roger 5880 is 2.2, Roger 4350 is 3.48, FR-4 is 4.4 and Roger 3010 is 10.2 .

\section{Percentage of FrequencyShifting of Different} Samples-Simulated result

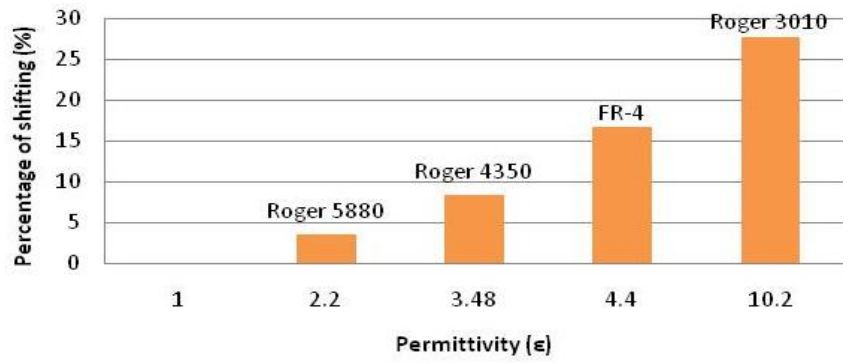

Figure 9. The percentage of frequency shifting of different MUTs

\subsection{Measured Result}

The proposed resonator sensor is fabricated on Roger 5880 substrate and it is found to be working on $3.98 \mathrm{GHz}$ of resonant frequency. Figure 10 shows the experimental setup for the fabricated sensor. The changes of resonant frequency and return loss of the MUTs are measured by using the vector Network Analyzer (VNA). The s1P data gained from the measurement is imported to the CST software. Based on the $\mathrm{S} 1,1$ result, it shows that the return loss is $-6.3575 \mathrm{~dB}$ and the Q-factor are 174 . The improvement of the sensor in term of Q-factor is $54 \%$.

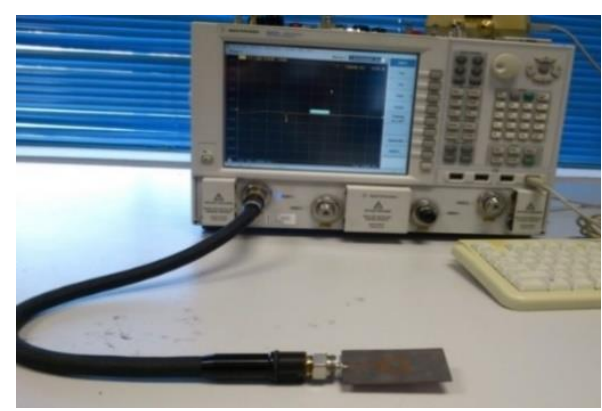

Figure 10. Experimental setup of the sensor 
While, Figure 11 shows the result of the measurement of different types of MUTs and permittivity. The data in Figure 11 is represented in the bar chart; Figure 12. The measurement results show that the sample of Roger 3010 has the highest percentage of frequency shifting with a large difference of frequency when compared with the resonant frequency without shifting. The percentage of frequency shifting is $26.7 \%$. The lowest percentage of frequency shifting happens with the sample Roger 5880, followed by Roger 4350 and FR-4. The measurement result also shows that the higher the permittivity of the sample, the larger the percentage of frequency shifting.

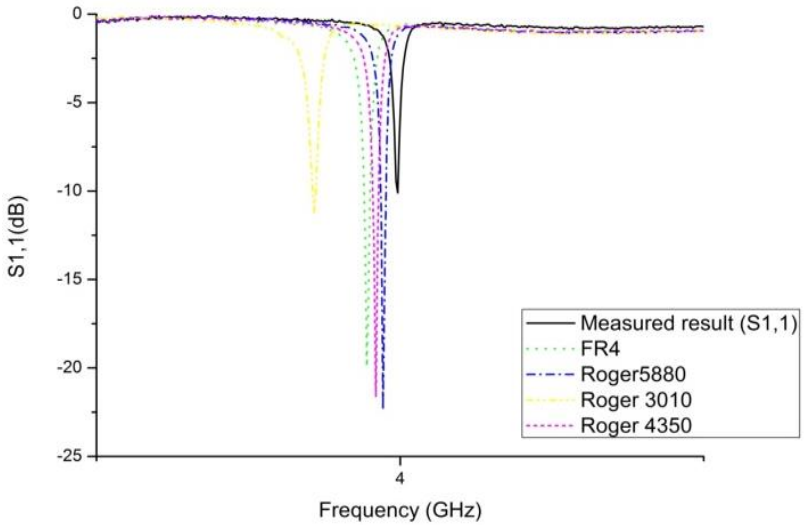

Figure 11. The changes of resonant frequency with different overlay samples in simulation

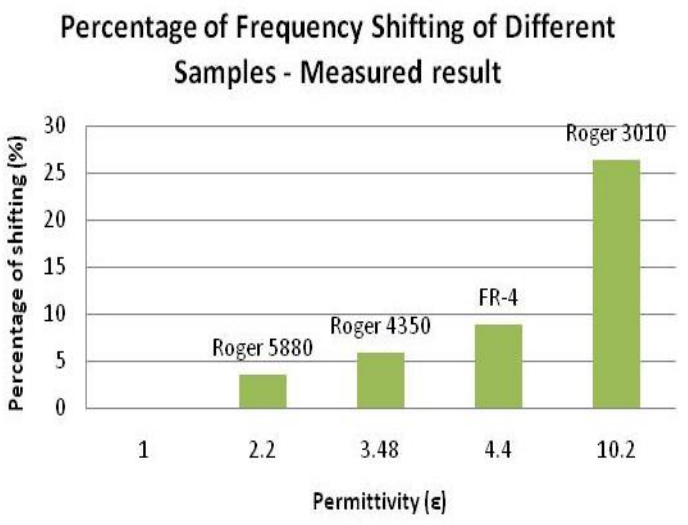

Figure 12. The percentage of frequency shifting of different MUTs

Then, the simulation and measurement results of the sensor without sample (air) are demonstrated in Figure 13. A small deviation occured between the simulated and measured results. The measured resonance frequency is slightly shifted from the simulation and the magnitude of the return loss is higher than the simulated results. This is due to the mismatch between the feed-line and the connector of the portand the tolerance of fabrications which limits the simulation accuracy.

The result shown in Figure 13 illustrates that the performance of the fabricated resonator sensor is in a good agreement with the sensor in the simulation. By using the measured data, the relationship between the shifting of resonant frequency and the standard permittivity can be modeled by using the second order polynomial. It is the derivation of numerical model which is used to calculate the permittivity of the MUT. The derivation model applied the curve fitting method instead; refer to Figure 14. Then, the measured permittivity of the tested MUT is mathematically expressed as Equation 2.

$$
\varepsilon_{\gamma}=-21.25 f(x)^{2}+128.5 f(x)-173.9
$$

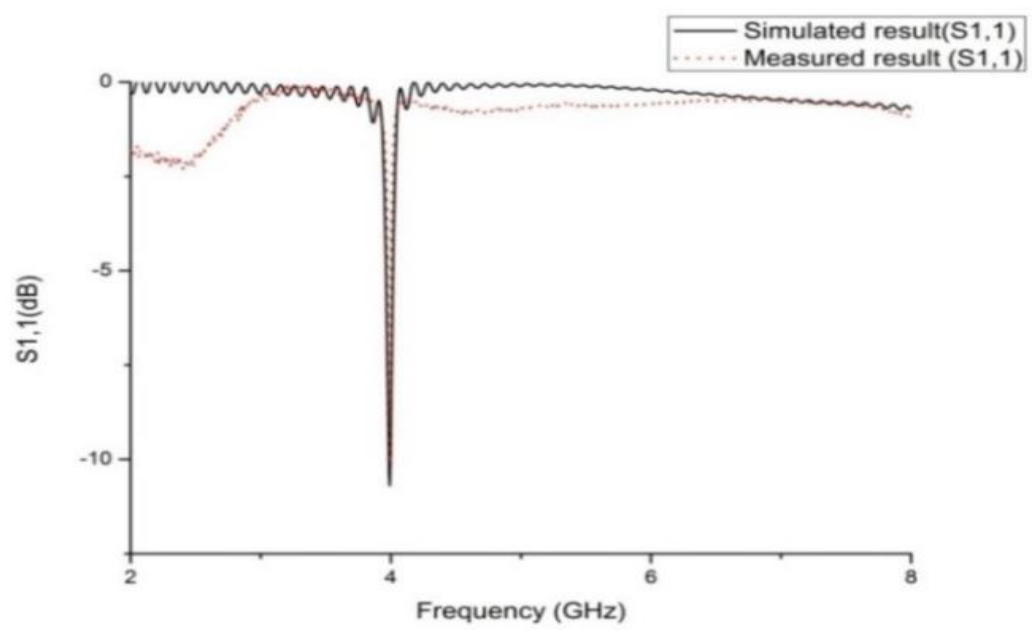

Figure 13. Comparison between simulated and measured results based on the $\mathrm{S} 1,1$ 


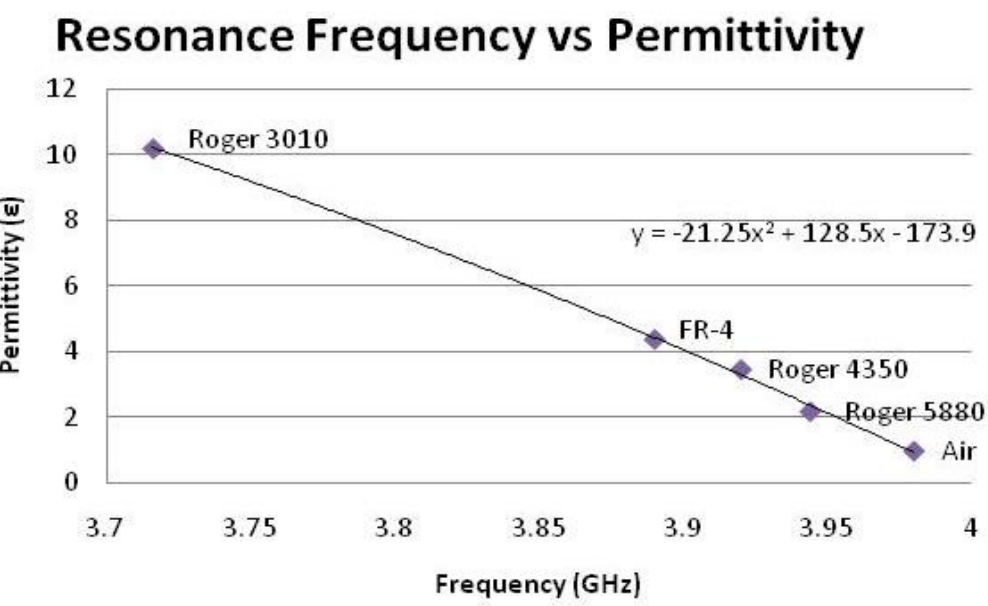

Figure 14. The relationship between the resonant frequency and the permittivity

From Table 2, it shows the result of the calculated permittivity. The error of the permittivity regarding the usage of Equation 19 is in the range of $0.2 \%$ to $8 \%$. The higher the permittivity of the sample effect the lower the error.

Table 2. The Calculated Permittivity based on Equation (2)

\begin{tabular}{|c|c|c|c|c|}
\hline MUTs & Frequency $(\mathrm{GHz})$ & Permittivity, $\varepsilon_{Y}$ & Calculated ${ }^{\varepsilon_{Y}}$ & Error $(\%)$ \\
\hline Air & 3.98 & 1 & 0.9215 & 7.85 \\
\hline Roger 5880 & 3.944 & 2.2 & 2.3574 & 7.15 \\
\hline Roger 4350 & 3.92 & 3.48 & 3.284 & 5.63 \\
\hline FR-4 & 3.89 & 4.4 & 4.41 & 0.23 \\
\hline Roger 3010 & 3.716 & 10.2 & 10.17 & 0.29 \\
\hline
\end{tabular}

\subsection{Compared with Existing Resonator Sensor}

Table 3 shows the tabulated data which have been compared with the proposed resonator sensor. The comparison is made based on the resonance frequency, Q-factor and the return loss. From the table, it shows that the proposed resonator sensor has the highest Q-factor compared to other two designs. But, the return loss of the proposed sensor is the lowest. The result of the proposed sensor is due to the usage of the enhancement method in the design where it introduces the "pushing effect" on the sensor. The method being used by [17] and [18] shows the opposite result compared to the proposed rectangular sensor where the sensor has a very low $\mathrm{Q}$-factor, $\approx 64$ but the return loss can achieve until $-22.34 \mathrm{~dB}$. Then, each of the methods has a different level of resonance frequency.

Table 3. Comparison between Different Techniques with the Proposed Sensor [17-18]

\begin{tabular}{cccc}
\hline \multirow{2}{*}{ Method } & \multicolumn{3}{c}{ Specifications } \\
\cline { 2 - 4 } & Frequency $(\mathrm{GHz})$ & Q-factor & $\mathrm{S}_{2,1} / \mathrm{S}_{1,1}(\mathrm{~dB})$ \\
\hline$[17]$ & 2.65 & 80 & -21.00 \\
{$[18]$} & 1.28 & $\approx 64$ & -22.34 \\
Proposed sensor & 3.98 & 174 & -6.3575 \\
\hline
\end{tabular}

The error percentage is calculated to measure the accuracy of the proposed rectangular sensor. The data is represented in Table 4. Through the tabulated data, it shows that the proposed sensor gains the lower range of error compared to the existing sensor. The percentage of error in the permittivity for the proposed sensor is $0.29 \%$ to $7.85 \%$, while for the existing sensor; the percentage of the error in the permittivity is $0.57 \%$ to $12.70 \%$ instead.

While analyzing Table 4, it shows that the percentage of error in the proposed sensor is decreased linearly. The higher the permittivity of the MUTs, the lower the percentage of error in the rectangular sensor. But, data for the existing sensor are taken from various sources. Thus, it can't be analyzed based on the 
linearity of the error. But, in the existing sensor, the higher error is gained when there is Roger 5880 that act as the sample while the lowest error is gained when the Roger 4350 acts as the sample. When there is no sample overlay on the sensor, the error is $8 \%$. For the FR-4 sample, the error of the permittivity is $5.68 \%$.

Table 4. Comparison Measurement Accuracy of the Proposed Sensor with the Existing Resonator Sensor [2, $18,19-20]$

\begin{tabular}{cccccc}
\hline MUTs & Standard. & \multicolumn{2}{c}{ Existing sensor } & \multicolumn{2}{c}{ Proposed sensor } \\
\cline { 3 - 6 } & permittivity, $\varepsilon_{\boldsymbol{Y}}$ & $\varepsilon_{\boldsymbol{Y}}$ & \% of error & $\varepsilon_{\boldsymbol{Y}}$ & \% of error \\
\hline Air & 1 & 1.08 & 8.0 & 0.9215 & 7.85 \\
Roger 5880 & 2.2 & 1.92 & 12.70 & 2.3574 & 7.15 \\
Roger 4350 & 3.48 & 3.5 & 0.57 & 3.284 & 5.63 \\
FR-4 & 4.4 & 4.15 & 5.68 & 4.41 & 0.23 \\
Roger 3010 & 10.2 & - & - & 10.17 & 0.29 \\
\hline
\end{tabular}

\section{CONCLUSION}

The rectangular resonator sensor with the enhancement method is proposed in this paper. The proposed sensor design able to improve the return loss of the basic rectangular resonator sensor; less than -10 $\mathrm{dB}$. Regarding the simulated result, it shows that the performance of the proposed sensor is in good agreement with the calculated result. While, by measuring the permittivity of the known samples, it is found that the percentage of error for the proposed sensor is from $0.2 \%$ to $8 \%$. The sensitivity and accuracy of the sensor has been analyzed in terms of Q-factor and return loss. Plus, it is noted that the shifting of the resonance frequency is affected by the permittivity of the sample. The higher the permittivity of the samples effect the lower the resonance frequency. Thus, the fabricated resonator sensor is suitable to be applied in the real life like in the food industry, agriculture and so on.

\section{ACKNOWLEDGEMENTS}

The writers would like to thank Faculty of Electronics and Computer Engineering (FKEKK), and Center for Research and Innovation Management (CRIM), Universiti Teknikal Malaysia Melaka (UTeM) for supportive in attaining the information and material in the development for this work. The appreciation also goes to the Ministry of Higher Education (MOHE) and the Government of Malaysia which funding this project under the research grant JURNAL/2018/FKEKK/Q00001 and PJP/2017/FKEKK/HI10/S01532. Finally, we also thank the nameless referees whose comments led to an enriched presentation of our project.

\section{REFERENCES}

[1] Horestani AK, Abbot D, Fumeaux C. Rotation Sensor Based on Horn-Shaped Split Ring Resonator. IEEE Sensors Journal. 2013;13 (8): 1-2.

[2] Lee CS, Yang CL. Complementary Split Ring Resonators for Measuring Dielectric Constant and Loss Tangents. IEEE Microwave and Wireless Components Letters. 2014; 24 (8): 563-565.

[3] Lee CS, Yang CL. Single Compound Complementary Split Ring Resonator for Simultaneously Measuring Permittivity and Thickness. 2014 IEEE MTT-S International Microwave Symposium (IMS2014); pp. 1-3.

[4] Zarifi MH, Daneshmand M. Non-Contact Liquid Sensing Using High Resolution Microwave Micro - strip Resonator. Microwave Symposium (IMS); 2015: 1-4.

[5] Boybay MS, Ramachi OM. Non-destructive Thickness Measurement Using Quasi-Static Resonators. 2013; IEEE Microwave and Wireless Components Letter; 23 (4): 217-219.

[6] Surrendar R, Jackson B. Dielectric Characterization using Microwave Resonator Sensor. International Journal of Research in Engineering \& Advanced Technology (IJREAT). 2013; 1 (3): 1-4.

[7] Waldron IJ. Ring Resonator Method for Dielectric Permittivity Measurement of Foams. PhD Thesis. Electrical and Computer Engineering of Worcester Polytechnic Institute; 2006.

[8] Yang, CL, Lee CS. Thickness and Permittivity Measurement in Multi-Layered Dielectric Structures Using Complementary Split-Ring Resonators. Institute of Electrical and Electronics Engineers (IEEE Sensor Journal). 2014; 14 (3): 695-700.

[9] Qureshi RM. Ring Resonator with Single Gap for Measurement of Dielectric Constants of Materials. Master Thesis. University of Gavle; 2013.

[10] Buyukozturk O, Yu TY. Ortega JA. A Methodology for Determining Complex Permittivity of Construction Materials Based on Transmission-only Coherent, Wide-Bandwidth Free-space Measurements. Elsevier Ltd., 2006; 349-359.

[11] Kumar A, Sharma A, Singh G. Measurement of Dielectric Constant and Loss Factor of the Dielectric Material at Microwave Frequencies. Progress in Electromagnetics Research (PIER 69), 2007; 47-54.

[12] Steele CW. A Non-resonant Perturbation Theory. IEEE Transactions on Microwave Theory and Techniques, 1966; 14 (2): $70-74$. 
[13] Nasserdine M, Mengu'e S, Bourcier C, Richalot E. Field Measurements within a Large Resonant Cavity Based on the Perturbation Theory. Progress in Electromagnetics Research B, 2014; 57: 1-20.

[14] Saeed S, Shafique MF, Byrne MB, Hunter IC. Planar Microwave Sensors for Complex Permittivity Characterization of Materials and Their Applications, Applied Measurement Systems, 2012; 320-347.

[15] Chenv LF, Ong CK, Neo CP, Varadan VV, Varadan VK. Microwave Electronics: Measurement and Materials Characterization. West Sussex: John Wiley \& Sons, Ltd. 2004: 125-140.

[16] Kajfez D. Q-factor Measurement with A Scalar Network Analyser. Institute of Electrical and Electronics Engineers (IEEE Sensor Journal), 2002; 142 (5) :1350-2417.

[17] Ansari MAH, Jha AK. Akhtar MJ. Design and Application of the CSRR Based Planar Sensor for Non-invasive Measurement of Complex Permittivity. Institute of Electrical and Electronics Engineers (IEEE-Sensor Journal). 2015; 15 (12): 7181-7189,

[18] Boybay MS, Omar MR. Material Characterization using Complementary Split-Ring Resonators. IEEE Transactions on Instrumentation and Measurement. 2012; 61(11): 3039-3046.

[19] Rusni IM, Ismail A, Alhawari AR, Hamidon MN, Yusof NA. An Aligned-gap and Centered-Gap Rectangular Multiple Split Ring Resonator for Dielectric Sensing Applications. Journal of Sensors. 2014; 14 (7): $13134-$ 13148.

[20] Alahnomi RA, Zakaria Z, Ruslan E, Mohd Bahar, Ab Rashid AA. S. R. High Sensitive Microwave Sensor Based on Symmetrical Split Ring Resonator for Material Characterization. Microwave and Optical Technology Letters. 2016; 58 (9): 2106 - 2110. 